\title{
Adaptación y validación de la versión hispana del Cuestionario de Estilo Emocional ${ }^{*}$
}

\author{
Adaptation and Validation of the Spanish Version \\ of the Emotional Style Questionnaire
}

Recibido: febrero 4 de 2010 | Revisado: junio 5 de 2010 | Aceptado: agosto 8 de 2010

\author{
LETICIA GUARINO** \\ Universidad Simón Bolívar, Caracas, Venezuela
}

Para citar este artículo. Guarino, L. (2011). Adaptación y validación de la versión hispana del Cuestionario de Estilo Emocional. Universitas Psychologica, 10(1), 197-209.

* Artículo de investigación en psicometría.

** Dpto. de Ciencia y Tecnología del Comportamiento. Edificio de Estudios Generales, 1er. Piso. Valle de Sartenejas, Aptdo. Postal 89.000 - A. Edo. Miranda. Venezuela. Tel.: 00582129063595 . Fax: 0058212 9063811.E-mail: lguarino@usb.ve
RESUMEN

El presente trabajo reporta los resultados de la adaptación al español y validación del Emotional Style Questionnaire (ESQ) conducida con una muestra de estudiantes universitarios venezo lanos. La escala original de 56 items (Roger \& Najarian, 1989; Roger \& Nesshoever, 1987) medía cuatro dimensiones de estilo emocional: Rumiación, Inhibición Emocional, Control de la Agresión y Control Benigno. Sin embargo, basados en la revisión de las escalas, Roger, Guarino y Olason (2000) propusieron una versión abreviada de 39 ítems, en la cual solo se mantuvieron las escalas de Rumiación e Inhibición Emocional. La versión hispana de esta escala, junto con otras medidas de personalidad y de salud, fue administrada a una muestra de 419 estudiantes venezolanos y los resultados mostraron una estructura factorial casi idéntica a la de la versión anglosajona, con adecuadas consistencias internas. Los estudios de validez concurrente y predictiva apoyan la estructura teórica de cada dimensión.

Palabras clave autor

Validación, escala de estilo emocional.

Palabras clave descriptores

Estudiantes universitarios, Venezuela, personalidad y emociones, conducta y

mecanismos de conducta.

\section{A B S T R A C T}

The present paper reports the results of the Spanish adaptation and validation of the Emotional Style Questionnaire conducted with a sample of Venezuelan university students. The original 56 items scale (Roger \& Najarian, 1989; Roger \& Nesshoever, 1987) measured four dimensions of emotional styles: rumination, emotional inhibition, aggresion control and benign control. However, based on the resivion of the scales, Roger, Guarino and Olason (2000) propused a shorter version of 39 items, where only the rumination and emotional inhibition scales were maintained. The Spanish version of this scale, jointly with other personality questionnaires and health vmeasures, was administered to a sample of 419 Venezuelan university students and results showed the structure of the scale to be nearly identical to the English version, with adequate internal consistency. The concurrent and predictive validation study supports the theoretical structure for each dimension.

Key words author

Validation, Emotional Style Scale.

Key words plus

College students, Venezuela, Personality and Emotions, Behavior and Behavior Mechanisms. 


\section{Introducción}

El constructo de control emocional define la tendencia de los individuos a inhibir la expresión de las respuestas emocionales y la primera versión de la escala (Emotional Control Questionnaire [ECQ], por sus siglas en inglés) desarrollada por Roger y Nesshoever (1987) surgió, precisamente, en el marco de la investigación sobre el rol de la personalidad y las diferencias individuales como moderadoras de la relación entre el estrés y la enfermedad. Esta escala fue propuesta en respuesta a las limitaciones que, para ese momento, otros instrumentos de medida del estilo de respuesta emocional habían mostrado (para una revisión exhaustiva, véanse Roger \& Nesshoever, 1987).

Los autores mencionados iniciaron la construcción y validación del ECQ, usando una muestra de 328 estudiantes de la Universidad de York (Inglaterra), quienes respondieron a un pool inicial de 53 ítems relacionados con la manera en la cual respondían emocionalmente a situaciones cotidianas de la vida. Haciendo uso del análisis factorial, por la vía del método de análisis de componentes principales y rotación de máxima verosimilitud, la escala finalmente quedó reducida a 40 ítems con cuatro factores, agrupados de la siguiente forma: 11 ítems saturando en el factor de Rumiación, 11 ítems en el factor de Control de la Agresión, 9 ítems para el factor Inhibición Emocional y 9 ítems para Control Benigno, respectivamente.

De acuerdo con Roger y Nesshoever (1987), la dimensión de Rumiación medía la tendencia de los individuos a mantenerse preocupados por eventos molestos ocurridos en el pasado. Por otro lado, la Inhibición Emocional se refería al "embotellamiento" o inhibición en la expresión de las emociones experimentadas. El factor de Control de la Agresión, claramente, agrupó a los ítems referidos al control de las respuestas agresivas; mientras que el Control Benigno estuvo básicamente referido a respuestas sobre el control de la impulsividad.

Esta estructura de cuatro factores fue confirmada con una muestra diferente de 279 estudiantes adultos del programa de universidad abierta de la Universidad de York. Los factores se comportaron de manera estadísticamente independiente y con valores de consistencia interna aceptables $(\mathrm{Ru}-$ miación $=0.86$; Inhibición Emocional $=0.77$; Control de la Agresión $=0.81 ;$ y Control Benigno $=0.79$, respectivamente). De igual forma, la fiablidad fue aceptable, tanto al cabo de 4 semanas como de 6 meses, oscilando entre 0.75 y 0.91 , respectivamente para ambos períodos, asumiendo los autores la estabilidad de las medidas (véanse Roger \& Nesshoever, 1987).

Con respecto al estudio de validez concurrente para este cuestionario, puede decirse que, en general, las escalas de Control de la Agresión y Control Benigno correlacionaron inversamente y en forma significativa con escalas de impulsividad. Por otro lado, la escala de Rumiación correlacionó significativamente con una escala de neuroticismo y a su vez con la escala de Inhibición Emocional.

En un estudio posterior, Roger y Najarian (1989) trabajaron en la expansión de las escalas del cuestionario, considerando la brevedad de las mismas como una de sus principales limitaciones (escalas con 9 ítems), las cuales no parecían explorar suficientemente el repertorio conductual asociado a los factores. Como resultado de este análisis, propusieron el ECQ2, cuestionario de 56 ítems que replicó la estructura de cuatro factores descrita anteriormente, pero conteniendo ahora 14 ítems en cada dimensión como resultado del nuevo análisis factorial. Los índices de fiabilidad y consistencia interna para esta nueva versión fueron bastante aceptables para todas las escalas (oscilando entre 0.73 y 0.92). El estudio de validez concurrente para este nuevo cuestionario indicó, al igual que el estudio anterior, que las dimensiones de Control de la Agresión y Control Benigno estaban correlacionadas con el factor de extraversión del modelo de personalidad de Eysenck (Eysenck Personality Inventory [EPI], Eysenck \& Eysenck, 1964). Más específicamente, el Control Benigno correlacionó inversamente con medidas bien establecidas de impulsividad (Eysenck \& Eysenck, 1978), mientras que el Control de la Agresión mostró estar asociado negativamente a problemas de manejo de la rabia en jóvenes delincuentes (McDougall, Venables \& Roger, 1991). 
Siguiendo con el análisis y depuración del instrumento, Roger, Guarino y Olason (2000) propusieron una readaptación del ECQ2, denominándolo en adelante Cuestionario de Estilo Emocional (Emotional Style Questionnaire [ESQ], por sus siglas en inglés). Dicho proceso consistió en expandir las escalas de Rumiación e Inhibición Emocional , así como en eliminar los ítems de las escalas de Control de la Agresión y Control Benigno, por considerar que éstas describían de manera importante otras esferas de personalidad como la impulsividad y el deseo de aventura (Eysenck \& Eysenck, 1978) y que no estaban directamente relacionas con el manejo de las emociones en el contexto del estudio del estrés y la enfermedad.

Dado que los ítems que formaban la escala de Rumiación describían básicamente la repetición y remembranza de eventos estresantes pasados, en esta nueva revisión de la escala se añadieron ítems que describían situaciones o eventos estresantes futuros (preocupación sobre eventos que pueden ocurrir). Por su parte, la escala de Inhibición Emocional fue expandida a través de la incorporación de algunos ítems provenientes de un cuestionario que mide la capacidad de usar el apoyo social, el Interpersonal Trust Questionnaire (ITQ) de Forbes y Roger (1999), básicamente de las subescalas de Miedo a la Revelación y Afrontamiento Social, por estar referidas a la expresión emocional.

Un análisis preliminar del nuevo conjunto de 67 ítems mostró una estructura claramente definida de dos factores, uno relacionado con la Rumiación (18 items) y el segundo describiendo a la Inhibición Emocional (21 ítems). Los índices de consistencia interna para ambas escalas fueron satisfactorios ( 0.875 y 0.905 , respectivamente) para una muestra de 174 estudiantes universitarios británicos (Roger et al., 2000). En el mismo estudio se analizó la validez predictiva del cuestionario con una muestra diferente de 218 estudiantes en proceso de adaptación a la universidad ( 84 hombres, edad media $=18.7, D T=1,48 ; 134$ mujeres, edad media $=19.1, D T=2,44)$, encontrándose que el factor de Rumiación predijo significativamente el deterioro de la salud de los jóvenes al cabo de ocho semanas, medido a través de índices como el CHIPS (Cohen-Hoberman Inventory of Physical Symptoms de Cohen \& Hoberman, 1983), el GHQ (General Health Questionnaire de Golberg \& Williams, 1988), el SWLS (Satisfaction With Life Scale de Diener, Emmons, Larsen \& Griffin, 1985), el PSS (Perceived Stress Scale de Cohen, Kamarck \& Mermelstein, 1983) y el HSI (Health Status Inventory de Meadows, 1989). La Inhibición Emocional también predijo algunos índices de deterioro, pero en una magnitud menor que la Rumiación.

El ESQ, en su versión original en inglés, fue empleado en estudios posteriores en la Unidad de Investigación del Estrés de la Universidad de York, en el marco de la investigación sobre el papel de las diferencias individuales y características de personalidad como moderadoras de la relación entre estrés y enfermedad, todos indicando el alto valor predictivo del instrumento para evaluar el estilo de respuesta emocional y su implicación en la salud (p. ej., Greco \& Roger, 2001; Guarino, 2004; Olason, 2001), especialmente la dimensión de Rumiación.

En consecuencia, y con el objeto de evaluar las características psicométricas de la versión adaptada de 39 ítems del ESQ a población hispano parlante, el cuestionario fue traducido y administrado a una muestra de estudiantes universitarios venezolanos, junto con otro grupo de cuestionarios que midieron características de personalidad e índices de salud. Este trabajo reporta precisamente los resultados de dicho análisis.

\section{Método}

\section{Muestra}

La muestra estuvo constituida por 419 estudiantes de primer año de la Universidad Simón Bolívar (Venezuela), todos cursantes de carreras en Ciencias Básicas y Tecnologías, quienes accedieron a participar voluntariamente en el estudio poco tiempo después de haber ingresado a la universidad. De ellos, 133 (32,2\%) eran mujeres ( $M$ edad $=17,42, \mathrm{DT}=1,28)$ y $280(67,8 \%)$ hombres $(\mathrm{M}$ edad $=17,64, D T=1,47)$. Seis de los participantes no informaron su edad y género, quedando la 
muestra válida final constituida por 413 participantes.

\section{Instrumentos}

Cuestionario de Estilo Emocional (Emotional Style Questionnaire [ESQ]): Roger, Guarino y Olason (2000)

La escala revisada de 39 ítems fue traducida al español por dos expertos bilingües y retraducida al inglés para comprobar la existencia de posibles discrepancias en la redacción de los ítems debidas a la traducción. Una vez resueltas las diferencias, el cuestionario fue aplicado manteniendo su escala de respuesta dicotómica original cierto-falso, con 21 para la medición de la dimensión de Inhibición Emocional y 18 para la medición de la Rumiación.

Para el estudio de validez concurrente se usaron los siguientes instrumentos:

Escala de Sensibilidad Emocional (ESE): Guarino y Roger (2005)

Esta escala mide la responsividad o reactividad emocional de los individuos a través de 45 ítems agrupados en tres dimensiones: (SEN) Sensibilidad Egocéntrica Negativa (22 ítems), (DE) Distanciamiento Emocional (10 ítems) y (SIP) Sensibilidad Interpersonal Positiva (13 ítems). La escala SEN describe la tendencia a reaccionar negativamente con emociones de aprehensión, rabia, desesperanza, vulnerabilidad y autocrítica ante los cambios ambientales y situaciones estresantes. Por su parte, la escala DE describe la tendencia de los individuos a evitar las reacciones emocionales negativas de los otros, manteniéndose distantes de aquellos que puedan estar experimentando circunstancias difíciles. Por último, la escala SIP describe la tendencia a orientarse emocionalmente hacia los otros, lo cual se caracteriza por la habilidad para reconocer con facilidad las emociones de otros y mostrar preocupación y compasión por aquellos que sufren o atraviesan circunstancias difíciles. Los índices test-retest para las tres dimensiones oscilaron entre 0,65 y 0,72 , mientras que las consistencias internas (coeficientes $\alpha$ ) se ubicaron en 0,824 para SEN; 0,742 para DE y 0,71 para SIP, respectivamente.

Índice de Reactividad Interpersonal (IRI): Davis (1980)

Para este estudio, se usaron las subescalas de Preocupación Empática (PE) y Distrés Personal (DP) del IRI, traducidas y adaptadas por la primera autora. Tales adaptaciones mostraron una alta correspondencia con las versiones al español propuestas por Pérez-Albéniz et al. (2003).

Para el estudio de validez predictiva, se usaron los siguientes indicadores de salud:

Inventario de Síntomas Físicos de CohenHoberman (CHIPS): Cohen y Hoberman (1983)

Este inventario presenta una lista de 33 síntomas físicos comunes, los cuales fueron cuidadosamente seleccionados por los autores con el objeto de excluir los síntomas de naturaleza obviamente psicológica (p.ej.: sentirse nervioso o deprimido). La escala incluye, sin embargo, muchos síntomas físicos que han sido tradicionalmente vistos como psicosomáticos (p.ej.: dolor de cabeza, pérdida de peso). Cada ítem fue calificado en función de "cuánto había molestado o estresado al individuo durante las últimas 5 semanas". Los ítems fueron calificados en una escala de 5 puntos desde: el problema no me ha molestado para nada hasta el problema ha sido en extremo molesto. La confiabilidad $\alpha$ de Cronbach para la versión hispana de la escala fue bastante aceptable $(\alpha=0,88 ; N=401)$.

\section{Lista de Chequeo del Estatus de Salud (Health} Status Checklist [HSC]): Meadows (1989)

Esta es una lista de chequeo de síntomas de 30 ítems, que fue desarrollada a partir de una extensa encuesta a médicos generales de Inglaterra, con el propósito de medir los síntomas más frecuentemente referidos por pacientes de consulta externa. Este instrumento fue adaptado y validado por la primera autora (véase Guarino, 2005) y mide dos dimensiones de salud: Síntomas Psicológicos y algunas 
Enfermedades Crónicas (10 ítems) y Síntomas Físicos y Agudos (7 ítems). Los índices de consistencia interna fueron bastante aceptables $(\alpha=0,81$ para Síntomas Psicológicos y Enfermedades Crónicas, y $\alpha=0,68$ para Síntomas Físicos y Agudos).

\section{Procedimiento}

Una vez recibidos los permisos de las diferentes instancias universitarias para administrar los instrumentos, los estudiantes fueron informados de los objetivos y alcances del estudio, por lo que en forma voluntaria procedieron a completar el ESQ junto con el resto de los cuestionarios, en grupos y en las mismas aulas de clase y horarios regulares de actividades.

\section{Resultados}

\section{Análisis Factorial}

Los datos fueron sometidos a análisis factorial exploratorio (AFE) usando el método de factorización de ejes principales del SPSS v. 12 y como criterio mínimo para extracción de ítems autovalores superiores a 2. El gráfico de sedimentación mostró claramente 2 factores. Asumiendo la relativa independencia de las dimensiones y la estructura factorial de la escala original en inglés, se llevó a cabo una solución terminal de dos factores usando la rotación ortogonal Varimax y 0.30 como criterio de saturación de los ítems.

Los resultados de la matriz rotada mostraron una estructura idéntica a la obtenida con la versión en inglés de Roger et al. (2000), con 21 ítems saturando en el Factor 1 y 18 saturando en el Factor 2. Los ítems que saturaron el factor 1 correspondieron todos a la dimensión de Inhibición Emocional (IE), encabezados por la afirmación "Me gusta hablar sobre los problemas, para sacármelos de la cabeza"; mientras que para el Factor 2 el ítem de mayor saturación fue "Si veo algo que me da miedo o irrita, la imagen permanece en mi cabeza durante mucho tiempo", describiendo la dimensión de Rumiación
(R). Cabe destacar que los ítems en cada factor saturaron bajo un patrón idéntico al de la versión en inglés, es decir, en el mismo orden y con pesos similares a la estructura original, comprobando la correspondencia de ambas escalas. En adelante, esta versión en español se denominará Cuestionario de Estilo Emocional (CEE) y se asumirá como medida válida del constructo de estilo emocional.

\section{Análisis de fiabilidad e intercorrelaciones entre factores}

Los índices de consistencia interna alfa de Cronbach fueron bastante aceptables para los dos factores, siendo $=0.85$ para (IE) Inhibición Emocional (21 ítems) y 0.82 para (R) Rumiación (18 ítems). La Tabla 1 muestra el cuestionario completo en su formato de autoadministración.

Tal como se esperaba, la escala de Rumiación correlacionó fuerte y positivamente con las dimensiones de Sensibilidad Egocéntrica Negativa (SEN) y Distrés Personal (PD), lo cual indica que la misma mide aspectos de elevada emocionalidad negativa. Tal patrón se repitió con las escalas de Distanciamiento Emocional (DE) y Preocupación Empática (PE), aunque con magnitudes mucho menores. Por otro lado, la correlación que resultó más alta para la escala de Inhibición Emocional fue aquella con el Distanciamiento Emocional (0.341), lo que sugiere que los individuos que presentan dificultades para expresar sus propias emociones demuestran igualmente dificultad para manejar la expresión emocional de los otros, por lo cual se alejan o distancian de ésta. Igualmente consistentes fueron las correlaciones halladas entre la Inhibición Emocional y la Sensibilidad Egocéntrica Negativa. En el caso de la correlación con las escalas de Sensibilidad Interpersonal Positiva y Preocupación Empática, respectivamente, los resultados confirmaron lo esperado, en el sentido de que los individuos que más tienden a inhibir su propia expresión emocional tendrán también mayores dificultades para comprender las emociones de los otros y empatizar con las mismas. 


\section{TABLA 1}

Cuestionario de Estilo Emocional (CEE)

Sexo: Edad:

Instrucciones: Lee las siguientes afirmaciones e indica cómo te sientes en relación con cada una encerrando en un círculo CIERTO o FALSO, según sea el caso. Si alguna de las afirmaciones no es enteramente cierta o falsa para ti, escoge la alternativa que más se te parezca. Si tú no has estado en la situación descrita, por favor indica como te sentirías si estuvieras en esa situación.

1. Yo recuerdo cosas que me molestan o enfurecen durante mucho tiempo.

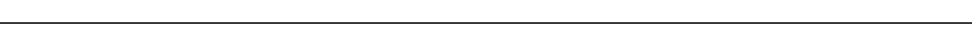

FALSO

2. Yo no soy una persona rencorosa: lo pasado, pasado está, y no pienso más en eso.

CIERTO

3. Cuando alguien me molesta, trato de ocultar mis sentimientos. FALSO

CIERTO

4. Algunas personas necesitan a alguien en quien confiar, pero yo prefiero resolver mis propios problemas.

FALSO

CIERTO

FALSO

5. Me agito solo de pensar en las cosas que me han irritado en el pasado.

CIERTO

FALSO

6. Frecuentemente me encuentro a mí mismo(a) pensando una y otra vez en las cosas típicas que me fastidian.

CIERTO

7. Aunque me sienta molesto(a) acerca de algo, no siento la necesidad de hablar con alguien sobre eso.

FALSO

8. A la gente le resulta difícil detectar en mí si yo estoy entusiasmado(a) o no con algo.

CIERTO

FALSO

9. Me gusta hablar sobre los problemas, para sacármelos de la cabeza.

CIERTO

FALSO

11. En el pasado se me ha hecho más fácil resolver los problemas, si he hablado sobre ellos con alguien.

12. Es bueno escuchar los problemas en voz alta.

CIERTO

FALSO

CIERTO

FALSO

CIERTO

FALSO

CIERTO

FALSO

13. Si recibo malas noticias delante de la gente, generalmente intento ocultar mis sentimientos.

CIERTO

FALSO

14. Yo siento que es útil hablar sobre los problemas, aun cuando ellos no tengan solución.

CIERTO

FALSO

15. Con frecuencia estoy preocupado(a) acerca de mi futuro.

CIERTO

FALSO

16. Tengo amigos que sé que me ayudarían, pero es difícil para mí pedir ayuda.

CIERTO

FALSO

17. Raramente muestro lo que siento acerca de las cosas.

CIERTO

FALSO

18. Si veo algo que me da miedo o irrita, la imagen permanece en mi cabeza durante mucho tiempo.

CIERTO

FALSO 
19. Yo pienso que la gente muestra sus sentimientos muy fácilmente.

CIERTO

FALSO

20. Mis fracasos me producen un constante arrepentimiento.

CIERTO

FALSO

21. Cuando algo me molesta prefiero hablarlo con alguien, más que aguantármelo por dentro.

CIERTO

FALSO

22. Para mí el futuro parece estar lleno de problemas e inconvenientes.

CIERTO

FALSO

23. Hay algunas situaciones en las cuales no me siento capaz de confiar en alguien.

CIERTO

FALSO

24. Con frecuencia siento como que estoy solo esperando que pase algo malo.

CIERTO

FALSO

25. Cuando alguien me recuerda mis fracasos pasados, siento como si estuvieran ocurriendo de nuevo

CIERTO otra vez.

FALSO

26. Si me pongo bravo(a) o me molesto, usualmente digo como me siento.

CIERTO

FALSO

27. A veces tengo que forzarme a mí mismo(a) a concentrarme en algo, para mantener los pensamientos CIERTO preocupantes sobre mi futuro fuera de mi mente.

FALSO

28. Los pensamientos intrusivos sobre problemas que tendré que enfrentar en el futuro me hacen difícil CIERTO concentrarme en una tarea.

FALSO

29. Yo no me siento avergonzado(a) de expresar mis sentimientos.

CIERTO

FALSO

30. Yo no dejo que un montón de cosas sin importancia me irriten.

CIERTO

FALSO

31. Desearía poder borrar de mi mente los recuerdos de fracasos pasados.

CIERTO

FALSO

32. No soy capaz de confiarle a alguien mis problemas.

CIERTO

FALSO

33. Tengo miedo de que si le confío mis problemas a alguien, se los cuente a otras personas.

CIERTO

FALSO

34. A veces pienso tanto en cosas que me molestan, que soy incapaz de sentirme positivo(a) acerca del futuro.

CIERTO

FALSO

35. No tengo miedo de pedir ayuda a alguien.

CIERTO

FALSO

36. Yo me preocupo mucho menos que otra gente acerca de cosas que podrían pasar.

37. Me toma un tiempo inusualmente largo olvidar cosas desagradables que me pasaron.

38. A veces no me siento capaz de confiar ni siquiera en alguien cercano a mí.

39. Cualquier cosa que me haga recordar mis experiencias desagradables me trae a la mente todas las 
Para el análisis de las intercorrelaciones entre las dimensiones del CEE, se calcularon correlaciones bivariadas de Pearson. Los resultados indicaron que las dimensiones de Rumiación (R) e Inhibición Emocional (IE) están ligeramente relacionadas $(r=0.265 ; p<0.001)$, replicando los resultados obtenidos previamente para diversas muestras anglosajonas de estudiantes universitarios (entre $r=0.157, p<0.05$; y $r=0.328, p<0.01$ ) para muestras de 177 y 61 estudiantes, respectivamente (véase Guarino, 2004).

\section{Validez concurrente}

Se evaluaron las asociaciones entre las dos dimensiones del CEE con cuestionarios que miden constructos relacionados, usando las tres escalas del ESE y las escalas de Preocupación Empática (PE) y Distrés Personal (DP) del IRI. Se calcularon correlaciones bivariadas usando la $r$ de Pearson y los resultados se muestran en la Tabla 2.

\section{TABLA 2}

Correlaciones entre las escalas del CEE y otras escalas

\begin{tabular}{lcc}
\hline \multicolumn{1}{c}{ Variables criterio } & $\begin{array}{c}\text { Rumiación } \\
(\mathrm{R})\end{array}$ & $\begin{array}{c}\text { Inhibición } \\
\text { Emocional (IE) }\end{array}$ \\
\hline $\begin{array}{l}\text { Sensibilidad Egocéntrica } \\
\text { Negativa (SEN) }\end{array}$ & $0.710 * *$ & $0.222 * *$ \\
$\begin{array}{l}\text { Sensibilidad Interpersonal } \\
\text { Positiva (SIP) }\end{array}$ & 0.027 & $-0.210 * *$ \\
$\begin{array}{l}\text { Distanciamiento Emocio- } \\
\text { nal (DE) }\end{array}$ & $0.285 * *$ & $0.341 * *$ \\
$\begin{array}{l}\text { Distrés personal } \\
\text { Preocupación Empática }\end{array}$ & $0.502 * *$ & $0.123 * * *$ \\
\hline$* * p<0.01$. & & $-0.211 * *$ \\
\hline $\begin{array}{l}\text { Fuente: elaboración propia. } \\
\text { Fen }\end{array}$ & & \\
\end{tabular}

\section{Estilo emocional y género}

Los estadísticos descriptivos para las dimensiones del CEE fueron calculados y sus resultados para la muestra total y por géneros se muestran en la Tabla 3
TABLA 3

Descriptivos para las escalas del CEE para la muestra total y por géneros

\begin{tabular}{lcc}
\hline & Rumiación & $\begin{array}{c}\text { Inhibición } \\
\text { Emocional }\end{array}$ \\
\hline Total $(\mathrm{N}=418)$ & $6.39(4.04)$ & $8.32(5.09)$ \\
Mombres $(\mathrm{N}=279)$ & $6.68(4.14)$ & $8.80(4.96)$ \\
Mujeres ( $\mathrm{N}=133)$ & $5.86(3.83)$ & $7.35(5.28)$ \\
Rangos observados & 0.17 & 0.20 \\
Rangos posibles & 0.18 & 0.21 \\
\hline
\end{tabular}

Fuente: elaboración propia.

Tal como se observa, los valores o puntuaciones totales para ambas dimensiones fueron relativamente bajos, tomando en cuenta las puntuaciones máximas posibles para cada una. Sin embargo, el grado de dispersión es elevado en cada caso, lo cual se corrobora con los valores mínimo y máximo observados.

La comparación por géneros usando el estadístico $t$ de Student, mostró que los hombres tienen significativamente más tendencia a la Rumiación $(t=1.94 ; g l=410 ; p=0.056)$ y a la Inhibición Emocional $(t=2.74 ; g l=410 ; p=0.006)$ que las mujeres, aunque esta diferencia fue significativa sólo para esta última dimensión. Ello sugiere que los hombres, al menos para esta muestra, tienen más dificultades para expresar sus emociones, por lo cual las inhiben o "embotellan".

\section{Validez Predictiva}

Con el objeto de determinar la capacidad predictiva de las dimensiones de Rumiación e Inhibición Emocional sobre indicadores de salud física y psicológica, se hizo una primera exploración del nivel de asociación usando la $r$ de Pearson. Seguidamente, se hicieron análisis de regresión múltiple usando el método de pasos sucesivos, para determinar si alguna de las dos dimensiones funcionaba como predictora de los índices de salud. 
La dimensión de Rumiación resultó positiva y significativamente asociada a los dos indicadores de síntomas físicos, tanto con la puntuación total del CHIPS $(r=0.336 ; p<0.0001)$, como con la subescala de Síntomas Físicos y Agudos del HSC $(r=0.142 ; p=0.004)$. Sin embargo, la Inhibición Emocional no resultó en absoluto asociada a este tipo de indicadores (CHIPS $-r=0.091 ; p<0.07$; HSC $-r=0.008 ; p=0.86$, respectivamente).

Para el caso de los síntomas psicológicos, los análisis fueron hechos separadamente por género debido a la constitución de la misma subescala, ya que se usaron versiones ligeramente diferentes para hombres y mujeres (véase Guarino, 2005). En este sentido, los resultados para los hombres indicaron que tanto la Rumiación como la Inhibición Emocional están positiva y significativamente asociadas al reporte de síntomas psicológicos, aunque la correlación es de mayor magnitud para la Rumiación $(r=0.38 ; p<0.0001$; Inhibición Emocional $r=$ $0.18 ; p=0.004)$. Por otro lado, los resultados para las mujeres fueron algo diferentes, ya que únicamente la Rumiación correlacionó positivamente y en forma significativa con el reporte de los síntomas psicológicos en este grupo $(r=0.42 ; p<0.0001)$, en contraste con la Inhibición Emocional $(r=$
$0.12 ; p<0.20)$. Lo anterior sugiere un mayor impacto negativo de ambos estilos emocionales sobre la salud psicológica de los hombres, mientras que para las mujeres la Rumiación parece ser el estilo emocional de mayor influencia negativa.

Los análisis de regresión múltiple, utilizando los indicadores de síntomas físicos y psicológicos, mostraron que únicamente la Rumiación fue capaz de predecir en forma significativa el deterioro en la salud de los estudiantes, con niveles de explicación de entre el 2 y el $17 \%$, tal como se muestra en las Tablas 4, 5 y 6. Específicamente, la Rumiación explica el $11 \%$ de las puntuaciones totales de los estudiantes en el cuestionario CHIPS, el cual evalúa presencia de síntomas físicos, indicando el impacto negativo que tiene este estilo emocional.

De igual forma, la Rumiación explica significativamente una proporción de la varianza de las puntuaciones totales en la subescala de Síntomas Físicos del HSC, aunque en un porcentaje mucho menor que para el inventario mencionado anteriormente (1,8\%). Esta discrepancia podría tal vez atribuirse a las características mismas del instrumento, lo cual requiere una discusión independiente a la del presente estudio.

\section{TABLA 4}

Análisis de regresión prediciendo puntuaciones en CHIPS

\begin{tabular}{lcccccc}
\hline & Beta & $T$ & $p$ & $R^{2}$ & $F$ & $g l$ \\
\hline $\begin{array}{l}\text { Variables en la ecuación } \\
\text { Rumiación }\end{array}$ & 0.336 & 7.133 & 0,0001 & 0.111 & $50.88^{* *}$ & 1,399 \\
\hline
\end{tabular}

$* * p<0,001$

Variable dependiente: CHIPS- Puntuaciones del Inventario Cohen-Hoberman

Fuente: elaboración propia.

\section{TABLA 5}

Análisis de regresión prediciendo puntuaciones en sub-escala de síntomas físicos

\begin{tabular}{|c|c|c|c|c|c|c|}
\hline & Beta & $T$ & $p$ & $R^{2}$ & F & $g l$ \\
\hline \multicolumn{7}{|c|}{ Variables en la ecuación } \\
\hline Rumiación & 0.142 & 2.878 & 0,004 & 0.018 & $8.28^{*}$ & 1,400 \\
\hline \multicolumn{7}{|c|}{$\begin{array}{l}* * p<0,01 \\
\text { Variable dependiente: Síntomas físicos del HSC. }\end{array}$} \\
\hline Fuente: elab & & & & & & \\
\hline
\end{tabular}


En el caso de los análisis para la subdimensión de Síntomas Psicológicos, tanto en hombres como en mujeres, nuevamente la única dimensión del CEE que explica las varianzas respectivas en la VD es la Rumiación, explicando en el caso de los hombres $14 \%$ y para las mujeres $17 \%$. Esto sugiere que la Rumiación parece tener efectos un poco más perturbadores para la salud mental de las mujeres, a diferencia de los hombres, dado su nivel de variabilidad explicada.

\section{Discusión}

Este trabajo presentó los resultados del proceso de adaptación al español y validación del Cuestionario de Estilo Emocional (CEE), versión abreviada del ECQ, propuesto inicialmente por Roger y cols. (Roger \& Najarian, 1989; Roger \& Nesshoever, 1987). Esta versión anglosajona de 39 ítems (ESQ) descrita por Roger, Guarino y Olason (2000), tuvo como objetivo la medición del estilo de respuesta emocional, concebido en dos dimensiones: Rumiación e Inhibición Emocional.

Dado que este cuestionario demostró adecuadas propiedades psicométricas y sobre todo una apropiada validez para predecir cambios en el estado de salud de los individuos sometidos a experiencias estresantes usando una muestra de estudiantes británicos, se consideró conveniente traducirlo, adaptarlo y validarlo al español para su uso en el contexto de la investigación del estrés, las diferencias individuales moderadoras y la salud en poblaciones hispano parlantes.
Los resultados del análisis factorial con la muestra de estudiantes venezolanos mostraron un patrón de saturación de los ítems idéntico al que había sido observado con la versión anglosajona (Roger et al., 2000), por lo que la escala en español quedó constituida por sus dos factores originales: Rumiación (18 ítems) e Inhibición Emocional (21 ítems). Los índices de consistencia interna fueron bastante aceptables, confirmando la confiabilidad de la escala. Por otro lado, las dimensiones resultaron ligeramente correlacionadas, replicando los hallazgos obtenidos con muestras anglosajonas (véase Guarino, 2004), tal como había sido observado en un estudio anterior, pero usando la versión completa del cuestionario traducido al español (56 ítems), en el cual también se obtuvo una correlación entre los dos factores (ver Roger et. al., 2001). Sin embargo, esta misma versión en español de 56 ítems con una muestra de 2816 personas de las Islas Canarias mostró resultados diferentes, dada la muy baja y no significativa correlación entre los dos factores (Matud, 2004).

Los resultados del estudio de validez concurrente fueron congruentes con lo esperado y apoyan la naturaleza del constructo. Específicamente, la Rumiación resultó positivamente correlacionada con medidas de emocionalidad negativa, como la Sensibilidad Egocéntrica Negativa del ESE (Guarino \& Roger, 2005) y la subescala de Distrés Personal del IRI (Davis, 1980). De igual forma, los resultados fueron consistentes para apoyar la validez del factor de Inhibición Emocional, el cual correlacionó positivamente con la subescala de Dis-

TABLA 6

Análisis de regresión para los síntomas psicológicos del HSC en hombres y mujeres

\begin{tabular}{|c|c|c|c|c|c|c|}
\hline & Beta & $T$ & $p$ & $R^{2}$ & F & $g l$ \\
\hline \multicolumn{7}{|c|}{ Variables en la ecuación para hombres } \\
\hline Rumiación & 0.375 & 6.516 & 0,0001 & 0.137 & $42.45 * *$ & 1,260 \\
\hline \multicolumn{7}{|c|}{ Variables en la ecuación para mujeres } \\
\hline Rumiación & 0.424 & 5.174 & 0,0001 & 0.173 & $26.768 * *$ & 1,122 \\
\hline \multicolumn{7}{|c|}{$\begin{array}{l}* * p<0,001 \\
\text { Variable dependiente: Síntomas psicológicos del HSC en hombres y mujeres. }\end{array}$} \\
\hline \multicolumn{7}{|c|}{ Fuente: elaboración propia. } \\
\hline 206 & JNIVERS & PYCHO & CA & No. 1 & ENERO-AB & | 2011 \\
\hline
\end{tabular}


tanciamiento Emocional del ESE, sugiriendo que aquellos individuos con dificultades para expresar sus propias emociones, también manifiestan una alta tendencia a evitar las expresiones emocionales de los otros, probablemente por la incapacidad para manejar la emocionalidad de éstos debida a la carencia de habilidades sociales e interpersonales.

La asociación positiva con la escala de sensibilidad egocéntrica negativa indica que los individuos que tienden a inhibir y no expresar sus emociones reaccionan en forma negativa ante los cambios y demandas ambientales, tal vez por la dificultad de manifestar su emocionalidad en forma más asertiva, empeorando aún más el patrón de reactividad. En el caso de la correlación con las escalas de Sensibilidad Interpersonal Positiva y Preocupación Empática, respectivamente, los resultados confirmaron lo esperado, en el sentido de que los individuos que más tienden a inhibir su propia expresión emocional, tendrán también mayores dificultades para comprender las emociones de los otros y empatizar con las mismas.

Esto último puede ser comprendido a la luz de los planteamientos que hacen Mayer, Caruso y Salovey (1999) sobre el modelo de inteligencia emocional, el cual asume que los seres humanos son entes procesadores de información, lo cual les permite responder de manera adaptativa ante el entorno. Dependiendo de esta capacidad, unas personas responderán mejor al medio que otras. Así, las personas con más inteligencia emocional son individuos que perciben de manera exacta la información de las emociones propias y de los demás, abstraen conceptos de estas percepciones para poder comprender las emociones y usan esta información para mejorar sus procesos cognitivos y para manejar sus emociones y las de otros individuos. La Inhibición Emocional, entonces, parecería ser un estilo de respuesta propio de las personas con dificultad para comprender y expresar tanto las emociones propias como las ajenas, y esta suposición debería examinarse analizando la posible asociación entre dicha variable y medidas de inteligencia emocional.

En relación a las diferencias entre géneros, los estudiantes de sexo masculino mostraron signifi- cativamente mayor Inhibición Emocional que las mujeres, lo que podría asumirse como esperado en una sociedad en la cual se promueve más la expresión emocional en las mujeres que en los hombres. Resultados similares encontró Matud (2004) usando el ECQ original de 56 ítems, a través del cual los hombres españoles de una comunidad de las Islas Canarias mostraron estadísticamente mayores niveles de Inhibición Emocional que las mujeres, no siendo esta diferencia significativa para el caso de la Rumiación. Sin embargo, los anteriores resultados contradicen lo reportado por Roger y cols. (p. ej. Roger \& Najarian, 1989; Roger \& Nesshoever, 1987), quienes no hallaron diferencias estadísticamente significativas entre géneros para ninguna de las dos dimensiones en muestras de estudiantes británicos, lo cual podría estar indicando una diferencia culturalmente determinada con respeto a la expresión emocional de los individuos.

Los análisis del estudio de validez predictiva usando los indicadores de síntomas físicos y psicológicos como variables dependientes, mostraron que únicamente la Rumiación fue capaz de predecir en forma significativa el deterioro en la salud de los estudiantes, con niveles de explicación entre 2 y $17 \%$. Sin embargo, el deterioro más acentuado se observó para la salud psicológica cuando los valores de Rumiación eran más elevados.

Estos resultados apoyan de manera consistente algunos hallazgos previos sobre la asociación entre la Rumiación y diferentes índices de adaptación fisiológica, como por ejemplo, en la recuperación demorada de la tasa cardiaca (Roger \& Jamieson, 1988) y en elevaciones sostenidas de cortisol en orina seguidas de la exposición a estímulos estresores (Roger, 1988; Roger \& Najarian, 1998). Se ha encontrado incluso que la Rumiación interactúa con eventos de vida negativos para predecir un mayor deterioro en la salud de estudiantes en período de adaptación a la universidad (Roger, 1995), así como con la escala de eventos positivos/ optimismo (PAO) del PANEQ (Olason \& Roger, 2001) en la predicción de depresión en muestras similares. De igual forma, la Rumiación mostró ser el predictor más fuerte de síntomas físicos y psicológicos, incluso después de haber sido controlados 
los valores iniciales de estas medidas tomadas seis semanas antes (Roger et al., 2000).

Usando la versión original del ECQ, Matud (2004) reportó resultados similares en el estudio con muestra de españoles, en el cual se hallaron correlaciones significativas entre Rumiación e indicadores de Estrés Crónico, Síntomas Somáticos y Distrés Psicológico. Sin embargo, García de la Banda, Martínez-Abascal, Riesco y Pérez (2004) no encontraron relación alguna entre los niveles de Rumiación, Inhibición Emocional y cortisol durante una experiencia estresante de examen con estudiantes universitarios españoles y tal como los autores mismos lo indican, estos resultados pudieran atribuirse más a fallas del diseño de investigación que al constructo mismo.

En definitiva, los resultados anteriores muestran que la versión hispana del ESQ (CEE en español) parece ser un instrumento válido y confiable para la medición de los estilos emocionales de los individuos y su uso podría considerarse muy valioso en el contexto de la investigación del estrés, en su relación con variables de personalidad, diferencias individuales y salud. En próximos estudios se sugiere confirmar la estructura factorial de esta versión en español, usando el AFC con muestras similares de hispano parlantes.

\section{Referencias}

Cohen, S., Kamarck, T. \& Mermelstein, R. (1983). A global measure of perceived stress. Journal of Stress and Social Behavior, 24, 385-396.

Cohen, S. \& Hoberman, G. M. (1983). Positive events and social support as buffers of life change stress. Journal of Applied Social Psychology, 13, 99-125.

Davis, M. H. (1980). A multidimensional approach to individual differences in empathy. JSAS Catalogue of Selected Documents in Psychology, 10, 85.

Diener, E., Emmons, R., Larsen, R. \& Griffin, S. (1985). The satisfaction with life scale. Journal of Personal Assessment, 49, 71-75.

Eysenck, H. J. \& Eysenck, S. B. G. (1964). Eysenck Personality Inventory. San Diego: Educational and Industrial Testing Service.
Eysenck, S. B. \& Eysenck, H. (1978). Impulsiveness and Venturesomeness: Their position in a dimensional system of personality description. Psychological Reports, 43, 1247-1255.

Forbes, A. \& Roger, D. (1999). Stress, social support and fear of disclosure. British Journal of Health Psychology, 4, 165-179.

García de la Banda, G., Martínez-Abascal, M. A., Riesco, M. \& Pérez, G. (2004). La respuesta de cortisol ante un examen y su relación con otros acontecimientos estresantes y con algunas características de personalidad. Psicothema, 16(2), 294-298.

Golberg, D. \& Williams, P. (1988). A user's guide to the General Health Questionnaire. Windsor, UK: NFER-Nelson.

Greco, V. \& Roger, D. (2001). Coping with uncertainty: The construction and validation of a new measure. Personality and Individual Differences, 31, 519-534.

Guarino, L. (2004). Emotional Sensitivity: A new measure of emotional lability and its moderating role in the stress-illness relationship. Tesis Doctoral sin publicar, Universidad de York, Inglaterra.

Guarino, L. (2005). Sensibilidad emocional. Su rol moderador en la relación estrés-enfermedad. Psicología Conductual, 13(2) 217-230.

Guarino, L. \& Roger, D. (2005). Construcción y validación de la Escala de Sensibilidad Emocional (ESE). Un nuevo enfoque para medir neuroticismo. Psicothema, 17(3), 465-470.

Matud, P. (2004). Gender diffferences in stress and coping styles. Personality and Individual Differences, 37, 1401-1415.

Mayer, J., Caruso, D. \& Salovey, P. (1999). Emotional intelligence meets traditional standards for an intelligence. Intelligence, 27(4), 267-298.

McDougall, C., Venables, P., \& Roger, D. (1991). Aggression, anger control and emotion control. Personality and Individual Differences, 12, 625-629.

Meadows, M. (1989). Personality, stress and health. Tesis Doctoral sin publicar, Universidad de York, Inglaterra.

Olason, D. (2001). Optimism, Pessimism and "Fighting spirit": Their role in moderating the stress response. Tesis Doctoral sin publicar, Universidad de York, Inglaterra. 
Olason, D. \& Roger, D. (2001). Optimism, pessimism and "fighting spirit": A new approach to assessing expectancy and adaptation. Personality and Individual Differences, 31, 755-768.

Pérez-Albéniz, A., de Paul, J., Etxeberría, J., Paz, M. \& Torres, E. (2003). Adaptación de Interpersonal Reactivity Index (IRI) al español. Psicothema, 15(2), 267-272.

Roger, D. (1988, julio). The role of emotion control in human stress responses. Comunicación presentada en la Conferencia Anual de la Sociedad Británica de Psicología, Universidad de Leeds, Inglaterra.

Roger, D. (1995, abril). The mechanics of stress: A model for the relationship between stress, health and personality. Comunicación presentada en la 5ta. Conferencia Internacional en Manejo del Estrés, Noordwijherhout, Holanda.

Roger, D., García de la Banda, G., Lee, H. S. \& Olason, D. T. (2001). A factor-analytic study of crosscultural differences in emotional rumination and emotional inhibition. Personality and Individual Differences, 31, 227-238.
Roger, D., Guarino, L. \& Olason, D. (2000, agosto). Emotional style and health: A new three-factor model. Comunicación presentada en la 14va. Conferencia Europea de Psicología de la Salud, Leiden, Holanda.

Roger, D. \& Jamieson, J. (1988). Individual differences in delayed heart-rate recovery following stress: The role of extraversion, neuroticism and emotional control. Personality and Individual Differences, 9(4), 721-726.

Roger, D. \& Najarian, B. (1989). The construction and validation of a new scale for measuring emotional control. Personality and Individual Differences, 10, 845-853.

Roger, D. \& Najarian, B. (1998). The relationship between emotional rumination and cortisol secretion under stress. Personality and Individual Differences, 24(4), 531-538.

Roger, D. \& Nessshoever, W. (1987). The construction and preliminary validation of a scale for measuring emotional control. Personality and Individual Differences, $8,527-534$. 
